\title{
PERFORMANCE ANALYSIS OF FAST AT FOR CORRIDOR AERIAL MAPPING
}

\author{
M. Blázquez, I. Colomina
}

Institute of Geomatics, Av. Carl Friedrich Gauss 11, Parc Mediterrani de la Tecnologia, Castelldefels, Spain marta.blazquez@ideg.es

KEY WORDS: INS/GNSS, sensor, orientation, method, aerial triangulation

\begin{abstract}
:
Nowadays most airborne photogrammetric and remote sensing systems are equipped with GNSS receivers and inertial sensors that allow the use of various orientation methods: from Direct Sensor Orientation (DiSO) to Integrated Sensor Orientation (ISO), all the way to "Fast Aerial Triangulation" (Fast AT), a new orientation method recently proposed by the authors of this paper. On the one hand, DiSO is the fastest method. It only needs the INS/GNSS time-position-attitude aerial control information and the previously calibrated system constants as the lever-arm vectors and boresight matrices. On the other hand, ISO is the most accurate and robust orientation and calibration method. It uses all the available information: a large number of image coordinates, some ground control points and the INS/GNSS time-position-attitude aerial control information. Fast AT is a new orientation method that combines the image measurement of few ground control points, the coordinates of these ground control points and the time-position-attitude aerial control information. Fast AT is clearly an alternative to DiSO, even an alternative to ISO depending on project specifications. Our preliminary results with regular blocks indicate that Fast AT performances are closer to the ISO than to the DiSO results. In the light of the good results for block aerial mapping, in this paper we investigate Fast AT performance for corridor aerial mapping: we briefly review the concept, discuss its geometry and analyse its potential for corridor aerial mapping.
\end{abstract}

\section{INTRODUCTION}

Today, photogrammetric and remote sensing sensors together with INS/GNSS equipment constitute the common data acquisition configuration for mapping systems. For this reason, Direct Sensor Orientation (DiSO) and Integrated Sensor Orientation (ISO), that use the time, position and attitude (tPA) INS/GNSS-derived information have consolidated as the standard methods for sensor orientation and calibration (Schwarz et al., 1993).

In the case of frame cameras, DiSO provides the sensor orientation parameters directly from the INS/GNSS-derived orientation parameters using the previously calibrated INS/GNSS lever arms and IMU-to-camera relative orientation (boresight) matrices. On the other hand, ISO provides the sensor orientation and calibration parameters performing a block adjustment using the INS/GNSS aerial control information, image coordinates and other available information as ground control points (GCPs). While DiSO is a direct orientation method, ISO is not only an orientation method, but also a sensor and system calibration procedure. DiSO is cheaper and faster than ISO, but ISO is more precise, accurate, robust and reliable (Heipke et al., 2002, Ip et al., 2007).

In (Blázquez and Colomina, 2012a) we propose a new orientation method, called "Fast Aerial Triangulation" (Fast AT) that sits halfway between DiSO and ISO. In fact, Fast AT is a particular case of ISO that allows us to orient the sensor and calibrate the system with less information. It consists of the use of image measurements of only few GCPs additionally to the INS/GNSS aerial control information. In general, Fast AT is more precise, accurate, robust, reliable and expensive than DiSO but less than ISO. In spite of the good results presented in (Blázquez and Colomina, 2012a), we do not claim that Fast AT replaces DiSO or ISO. It is just a new orientation method that depending on the project specifications can be a good alternative to ISO, for example, if image matching is difficult because of the ill-textured areas, or a good alternative to DiSO if some GCPs are available. Because of the excellent results that validate the Fast AT for block aerial mapping in (Blázquez and Colomina, 2012a), we investigate here the use of Fast AT for the corridor mapping application where the geometry is weaker.
In the next section, we review the concept and geometry of the Fast AT method. In the performance analysis section, the test data, the Fast AT corridor geometries and the results are discussed. Last, we present the conclusions of the perfomance of Fast AT for corridor aerial mapping.

\section{FAST AT}

Fast AT is a particular case of ISO characterized by the use of INS/GNSS-derived position and attitude aerial control, the coordinates of few GCPs and its corresponding image coordinates. All the tie points (TPs) are GCPs. The idea consists of using less information than in the classical ISO procedure.

The concept of Fast AT orientation method is independent to the mathematical models and the block adjustment software. But as an ISO procedure, we perform absolute Fast AT or relative Fast AT, in the same way that we perform absolute ISO or relative ISO (Blázquez and Colomina, 2012b). The traditional use of the tPA aerial control information is called absolute ISO. In this case, additionally to the orientation and calibration sensor parameters, we also estimate the calibration system parameters: GNSS linear shift (3D vector parameter that absorbs INS/GNSS errors) and boresight matrix. In (Blázquez and Colomina, 2012b) the proposed relative use of the tPA aerial control information that avoids estimating the GNSS linear shifts and the boresight matrix is called relative ISO. Therefore, absolute Fast AT is the ISO procedure where we use INS/GNSS-derived information in absolute mode, the coordinates of few GCPs and the image coordinates of these GCPs. In this case, we do not only compute the exterior orientation parameters, but also estimate the GNSS linear shift and boresight matrix parameters. On the other hand, relative Fast AT is the ISO procedure where we use INS/GNSS-derived information in relative mode, the coordinates of few GCPs and the image coordinates of these GCPs. In this case, we only estimate the exterior orientation parameters and forget about the system calibration parameters (the GNSS linear shifts and boresight matrix). Table 1 summarizes these main characteristics of DiSO, ISO and Fast AT orientation methods in terms of observables and estimable parameters. 


\begin{tabular}{|c|c|c|c|c|c|c|c|c|}
\hline & \multicolumn{3}{|c|}{ Observations } & \multicolumn{5}{|c|}{ Parameters } \\
\hline & ic & GCPs & $\begin{array}{l}\text { aerial } \\
\text { control }\end{array}$ & EOs & TPs & $\begin{array}{c}\text { Sensor } \\
\text { calibration }\end{array}$ & $\begin{array}{c}\text { GNSS } \\
\text { shifts }\end{array}$ & $\begin{array}{c}\text { Boresight } \\
\text { matrix }\end{array}$ \\
\hline DiSO & no & no & absolute & yes & no & no & no & known \\
\hline Absolute Fast AT & only for GCPs & few & absolute & yes & only the GCPs & no & $1 \times$ sub-block & yes \\
\hline Relative Fast AT & only for GCPs & few & relative & yes & only the GCPs & no & no & no \\
\hline Absolute ISO & many & few & absolute & yes & many & yes & $1 \times$ sub-block & yes \\
\hline Relative ISO & many & few & relative & yes & many & yes & no & no \\
\hline
\end{tabular}

ic: image coordinates; EOs: exterior orientation parameters.

Table 1: Observables and estimable parameters for DiSO, Fast AT and ISO procedures.

The Fast AT geometry is a function of the number, quality and distribution of observations, of the mathematical models in use and of the number and distribution of unknown parameters. Related to the unknown parameters, in (Blázquez and Colomina, 2012a) we define a sub-block as the set of images that share a common GNSS linear shift, if the INS/GNSS-derived data is processed in absolute mode. Equivalenty, if the INS/GNSS-derived data is processed in relative mode, a sub-block is defined as the set of images that are interconnected with relative aerial control observations. Both definitions are consistent. Related to the observations, in (Blázquez and Colomina, 2012a) we propose to use of redundant GCPs at the ends of each sub-block and to measure these GCPs in as many images as possible. Therefore, we characterize the Fast AT geometry by the number of sub-blocks, the number of strips per sub-block and the number of images per strip, the number of GCPs per sub-block and the number of images where a GCP is observed.

\section{PERFORMANCE ANALYSIS}

We analyse the performance of Fast AT, absolute Fast AT and relative Fast AT, through the horizontal and vertical ground accuracy and compare the results with those of DiSO and ISO, absolute ISO and relative ISO. The main indicator of our analysis is the Root Mean Square Error (RMSE) of check point (ChP) coordinate differences as compared to their pre-surveyed reference values. We compute the RMSE of ChPs in horizontal component, RMSE-H or $R_{H}$, and vertical component, RMSE-V or $R_{V}$, as:

$$
R_{H}^{2}=R_{E}^{2}+R_{N}^{2}, \quad \text { and } \quad R_{V}=R_{h}
$$

where $R_{E}, R_{N}$ and $R_{h}$ are the RMSE of ChPs in the east, north and height components, respectively.

To simplify the analysis of the RMSE of ChPs obtained by different blocks, aerial control modes (absolute and relative), orientation methods (DiSO, Fast AT and ISO) and components (horizontal and vertical), we define the improvement factor for Fast AT with respect to DiSO as

$$
\alpha=1-\frac{R^{\text {Fast } A T}-R^{I S O}}{R^{D i S O}-R^{I S O}} \quad\left(\text { for } R^{D i S O}>R^{I S O}\right),
$$

where $R^{I S O}, R^{F a s t A T}$ and $R^{D i S O}$ are the ground accuracy measures, horizontal or vertical, for the ISO, Fast AT and DiSO methods, respectively. An improvement factor close to $0 \%$ means that Fast AT performs like DiSO, an improvement factor close to $100 \%$ means that Fast AT performs like ISO and an improvement factor larger than $100 \%$ means that Fast AT performs better than ISO.

Additionally, as it was pointed in (Blázquez and Colomina, 2012a), we analyse the $r_{E}, r_{N}$ and $r_{h}$ corresponding to the GCP redundancy numbers in the east, north and height components, respectively. These values are correlated with the ground accuracy results and provide an indication of weakness in the Fast AT geometry.

\begin{tabular}{lrr} 
Test block & \multicolumn{1}{c}{ P-C } & \multicolumn{1}{c}{ S-C } \\
\hline Equipment & Leica RC30 & Z/I DMC \\
& Applanix & Applanix \\
& POS AV 510 & POS AV 510 \\
Image size & $23 \mathrm{~cm} \times 23 \mathrm{~cm}$ & $9 \times 17 \mathrm{~cm}$ \\
& $16329 \times 16329 \mathrm{px}$ & $7680 \times 13824 \mathrm{px}$ \\
Image size (along) & $23 \mathrm{~cm}$ & $9 \mathrm{~cm}$ \\
Image size (across) & $23 \mathrm{~cm}$ & $17 \mathrm{~cm}$ \\
Pixel size & $14 \mu \mathrm{m}$ & $12 \mu \mathrm{m}$ \\
Camera constant & $153 \mathrm{~mm}$ & $120 \mathrm{~mm}$ \\
Flying height $(\approx)$ & $1200 \mathrm{~m}$ & $1000 \mathrm{~m}$ \\
Scale $(\approx)$ & $1: 8000$ & $1: 8800$ \\
GSD $(\approx)$ & $11 \mathrm{~cm}$ & $11 \mathrm{~cm}$ \\
Strips & $3(1+2)$ & $3(1+2)$ \\
Images & 31 & 37 \\
Ground Check Points & 12 & 6 \\
Overlap $(\approx)$ & $60 \%$ & $60 \%$ \\
\hline
\end{tabular}

Table 2: Test blocks: geometric configuration.

\begin{tabular}{cc|cc|cc}
$\begin{array}{c}\text { Test } \\
\text { block }\end{array}$ & $\begin{array}{c}\text { No. of } \\
\text { GCPs }\end{array}$ & $\begin{array}{c}\text { No. of } \\
\text { TPs }\end{array}$ & $\begin{array}{c}\text { No. of } \\
\text { ic }\end{array}$ & \multicolumn{2}{c}{ Fost AT } \\
TPs & $\begin{array}{c}\text { No. of } \\
\text { ic }\end{array}$ \\
\hline \multirow{3}{*}{ P-C } & 4 & 282 & $933 \times 2$ & 4 & $12 \times 2$ \\
& 5 & 282 & $933 \times 2$ & 5 & $18 \times 2$ \\
& 7 & 282 & $933 \times 2$ & 7 & $28 \times 2$ \\
\hline \multirow{3}{*}{ S-C } & 4 & 610 & $2023 \times 2$ & 4 & $12 \times 2$ \\
& 6 & 610 & $2023 \times 2$ & 6 & $22 \times 2$ \\
& 8 & 610 & $2023 \times 2$ & 8 & $33 \times 2$ \\
\hline
\end{tabular}

No.: number; ic: image coordinates.

Table 3: Test blocks: number of GCPs, image coordinate observations and TPs.

\subsection{Test data}

This paper is focused in the performance analysis of Fast AT for corridor aerial mapping. Therefore, from two available blocks: Pavia block (P) and Salou block (S) (Blázquez and Colomina, 2012b), we generate two corridors: Pavia corridor (P-C) and Salou corridor (S-C), respectively. The Pavia corridor was captured with an analogue Leica RC30 camera and the Salou corridor was captured with a digital large format Zeiss/Intergraph (Z/I) DMC (DMC1-0026) camera. Details on the geometric configuration of the corridors are given in Table 2. Table 3 provides the number of image coordinate observations and TPs for ISO and Fast AT methods, depending on the number of GCPs. Figure 1 and Figure 2 represent the general layout of these corridors and Table 4 details the precision of their observations.

\subsection{Fast AT configurations}

In (Blázquez and Colomina, 2012a) and the previous section 2, we discuss the Fast AT geometry. This geometry depends on the 


\begin{tabular}{lrrl} 
& \multicolumn{2}{c}{ Block } & \\
Observables & P-C & S-C & Units \\
\hline Image coordinates & & & \\
- $\sigma_{x, y}$ & 4.8 & 1.5 & $\mu \mathrm{m}$ \\
& 0.34 & 0.13 & $\mathrm{px}$ \\
Ground Control Points & & & \\
- $\sigma_{E, N}$ & 8 & 5 & $\mathrm{~cm}$ \\
- $\sigma_{h}$ & 10 & 6 & $\mathrm{~cm}$ \\
Position absolute aerial control & & & \\
- $\sigma_{E, N}$ & 3.5 & 3.5 & $\mathrm{~cm}$ \\
- $\sigma_{h}$ & 5.5 & 5.5 & $\mathrm{~cm}$ \\
Attitude absolute aerial control & & & \\
- $\sigma_{\gamma, \theta}$ & 5 & 5 & $\mathrm{mdeg}$ \\
- $\sigma_{\psi}$ & 8 & 8 & $\mathrm{mdeg}$ \\
Position relative aerial control & & & \\
- $\sigma_{\Delta E, \Delta N}$ (within strips) & 4 & 4 & $\mathrm{~mm}$ \\
- $\sigma_{\Delta h}$ (within strips) & 6 & 6 & $\mathrm{~mm}$ \\
- $\sigma_{\Delta E, \Delta N}$ (between strips) & 3.5 & 3.5 & $\mathrm{~cm}$ \\
- $\sigma_{\Delta h}$ (between strips) & 5.5 & 5.5 & $\mathrm{~cm}$ \\
Attitude relative aerial control & & & \\
- $\sigma_{\gamma, \theta}$ (average within strips) & 1 & 1 & $\mathrm{mdeg}$ \\
- $\sigma_{\psi}$ (average within strips) & 1 & 1 & $\mathrm{mdeg}$ \\
- $\sigma_{\gamma, \theta}$ (average between strips) & 5 & 4 & $\mathrm{mdeg}$ \\
$-\sigma_{\psi}$ (average between strips) & 8 & 6 & $\mathrm{mdeg}$ \\
\hline \multicolumn{2}{l}{ Table }
\end{tabular}

Table 4: Test blocks: precision of observables.

number of images, strips, sub-blocks, GCPs and image observations of these GCPs. Because of the available Pavia and Salou block data, the Pavia and Salou corridors are composed by 3 flight lines. In the interest areas, there are 7 and 8 available GCPs for Pavia and Salou corridor, respectively. Therefore, the three possible Fast AT geometries of Pavia corridor and Salou corridor are represented in Figure 1 and Figure 2, respectively.

In the case of Pavia corridor, the first flight line is the vertical-left one, FL2, the second flight line is the horizontal one, FL3, and the last flight line is the vertical-right one, FL1. The proposed Fast AT geometries for Pavia corridor are:

- 1 sub-block and 4 GCPS. For absolute Fast AT, it is 1 GNSS linear shift for all images. For relative Fast AT, it is relative orientation interconnecting images of FL2, FL3 and FL1. The GCP configuration is 2 GCPs at the beginning of FL2 and 2 GCPs at the end of FL1.

- 2 sub-blocks and 5 GCPs. For absolute Fast AT, it is 2 GNSS linear shifts: one for the images of FL2 and FL3 and another one for the images of FL1. For relative Fast AT, it is relative orientation interconnecting images of FL2 and FL3. The GCP configuration is 2 GCPs at the beginning of FL2, 1 GCPs in the intersection of FL3 and FL1 (there is only one available GCP in this area) and 2 GCPs at the end of FL1.

- 3 sub-blocks and 7 GCPs. For absolute Fast AT, it is 3 GNSS linear shifts: one for each flight line. For relative Fast AT, this means that there is not relative orientation interconnecting images of different flight lines. The GCP configuration is 2 GCPs at the beginning of FL2, 2 GCP in the intersection of FL2 and FL3, 1 GPCs in the intersection of FL3 and FL1 and 2 GPCs at the end of FL1.

In the case of Salou corridor, the first flight line is the oblique one, FL7, the second flight line is the horizontal one, FL5, and the last flown flight line is the vertical one, FL6. The proposed Fast AT geometries for Salou corridor are:

- 1 sub-block and 4 GCPs. For absolute Fast AT, it is 1 GNSS linear shift for all images. For relative Fast AT, it is relative orientation interconnecting images of FL7, FL5 and FL6. The GCP configuration is 2 GPCs at the beginning of FL7 and 2 GCPs at the end of FL6.

- 2 sub-blocks and 6 GCPs. For absolute Fast AT, it is 2 GNSS linear shifts: one for the images of FL7 and FL5 and another one for the images of FL6. For relative Fast AT, it is relative orientation interconnecting images of FL7 and FL5. The GCP configuration is 2 GCPs at the beginning of FL7, 2 GCPs in the intersection of FL5 and FL6 and 2 GCPs at the end of FL6.

- 3 sub-blocks and 8 GCPs. For absolute Fast AT, it is 3 GNSS linear shifts: one for each flight line. For relative Fast AT, this means that there is not relative orientation interconnecting images of different flight lines. The GCP configuration is 2GCPs at the beginning of FL7, 2 GCPs in the intersection of FL7 and FL5, 2 GCPs in the intersection of FL5 and FL6 and 2GCPs at the end of FL6.

In order to determine which previous proposed geometry is more appropiate for Pavia and Salou corridors, we need to stablish the number of sub-blocks of each corridor. In terms of RMSE of $\mathrm{ChPs}$ for horizontal and vertical components, Figure 3 provides the absolute and relative ISO performances of the nine geometries that result from combining the three sub-block configurations by the three GCP configurations. According to the results of this figure, the best geometry of the Pavia corridor is a 3 sub-block corridor, independently of the GCP configuration, and the best geometries of the Salou corridor are a 2 sub-block or a 3 subblock corridor, independently of the GCP configuration. In the case of Salou corridor, even for ISO, some GCPs in the intersections of different flight lines are advisable.

\subsection{Fast AT results for corridor aerial mapping}

After the discussion of the previous section 3.2, the selected Fast AT geometry for Pavia corridor is a 3 sub-block configuration and 7 GCPs distributed at the ends of the sub-blocks. On the other hand, in order to minimize the number of estimated parameters, the selected Fast AT geometry for Salou corridor is a 2 sub-block configuration, instead of the possible 3 sub-block configuration. In this case, the GCP configuration is 6 GCPs distributed at the ends of these 2 sub-blocks. Table 5 presents the results of these selected Fast AT geometries in terms of RMSE of ChPs for horizontal and vertical components compared to DiSO and ISO performance of the same geometries. From the results of this table, if we compute the improvement factors for absolute and relative Fast AT with respect to DiSO of the Pavia and Salou corridors in horizontal and vertical components, we realize that Fast AT performance improves $74 \%$ with respect to DiSO performance in average. For components, in the horizontal component the average improvement factor is $80 \%$ and in the vertical component the average improvement factor is $68 \%$. On the other hand, the average improvement factor for absolute Fast AT is $69 \%$ while the average improvement factor for relative Fast AT with respect to DiSO is $79 \%$.

Finally, although we only present the ground accuracy results for the selected Pavia and Salou corridor geometries to compare the Fast AT method with the DiSO and ISO methods, the nine geometries as the result of combining the three sub-block configuration and the three GPC configuration were performed for absolute and relative Fast AT. In (Blázquez and Colomina, 2012a), we introduce the idea that the GCP redundancy numbers could be used as a self-diagnosis tool to detect a weak Fast AT geometry. Table 6 provides the GCP redundancy numbers for all the performed tests to check this idea for the presented corridors. According to these 
1 sub-block and 4 GCPs

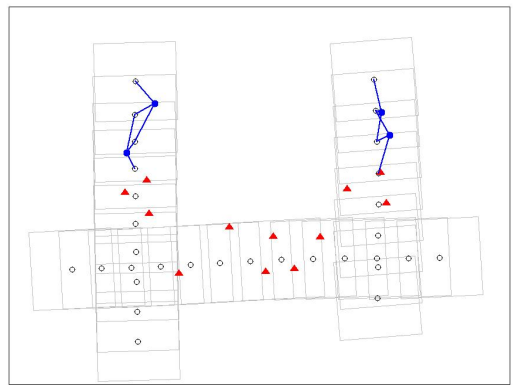

2 sub-blocks and 5 GCPs

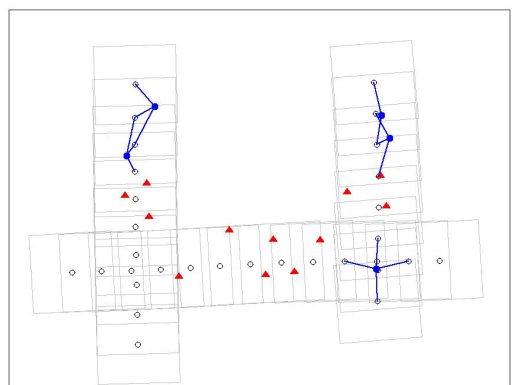

3 sub-blocks and 7 GCPs

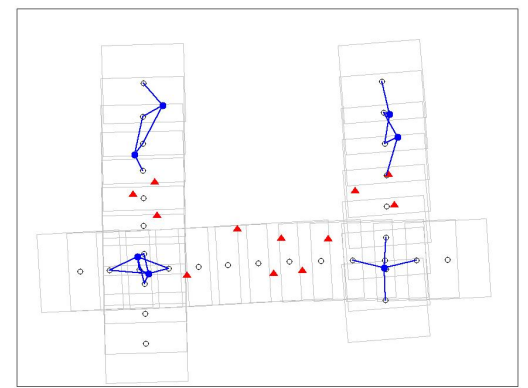

$\circ$ images $\quad \bullet \quad 0$ photo-measurements $\quad$ ground control points $\Delta$ check points

Figure 1: Pavia corridor: sub-block and GCP configurations.

1 sub-block and 4 GCPs



2 sub-blocks and 6 GCPs

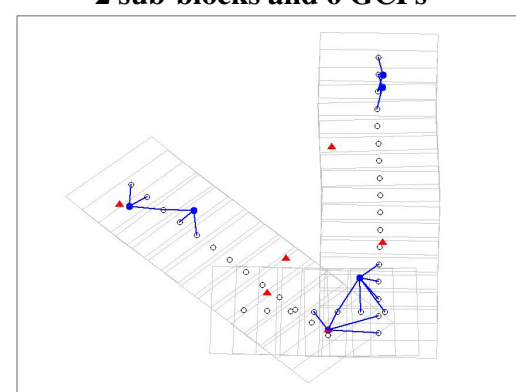

3 sub-blocks and 8 GCPs

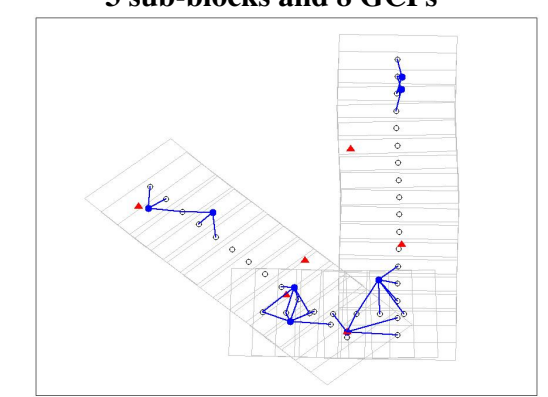

$\circ$ images $\quad \bullet$ photo-measurements $\quad$ ground control points $\Delta$ check points

Figure 2: Salou corridor: sub-block and GCP configurations.

Absolute ISO of Pavia Corridor

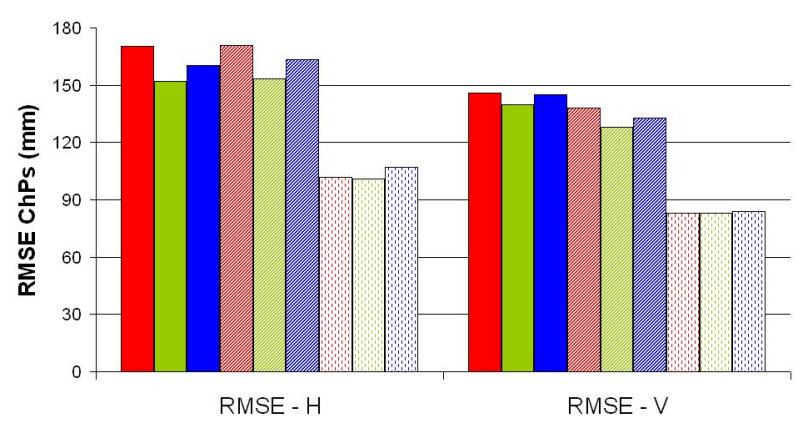

口 1sb+4gcp $\square$ 1sb+5gcp 1sb+7gcp 圈 2sb+4gcp 圆 2sb+5gcp 国 $2 s b+7 g c p$ 圆3sb+4gcp 圆3sb+5gcp 国 $3 s b+7 g c p$

Absolute ISO of Salou Corridor

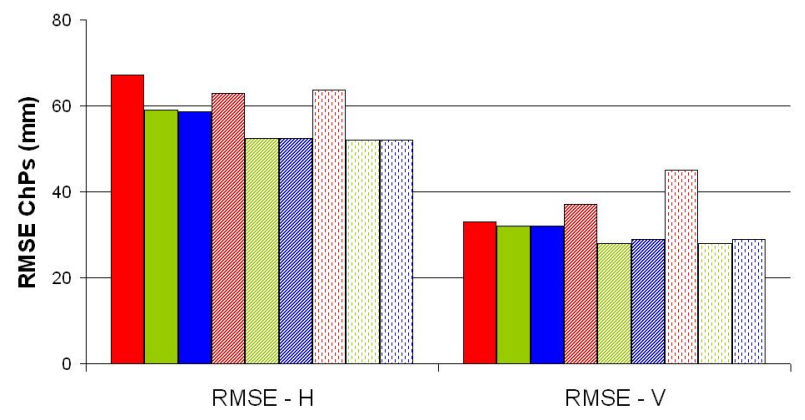

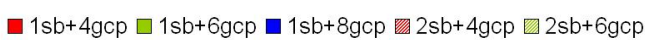

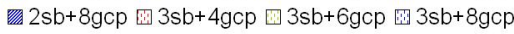

\section{Relative ISO of Pavia Corridor}

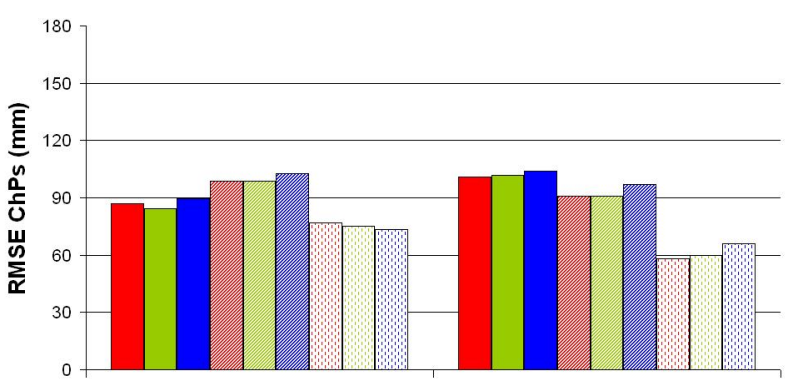

RMSE - H

RMSE - $V$

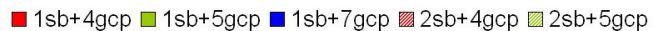
2sb+7gcp 圆3sb+4gcp 四3sb+5gcp 웅 3sb+7gcp

Relative ISO of Salou Corridor

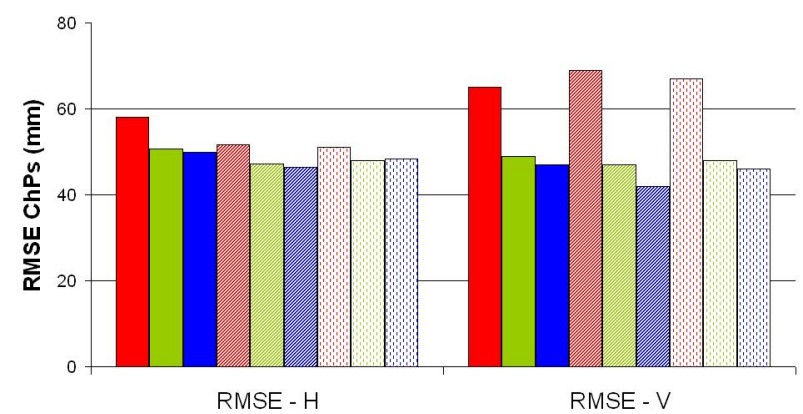

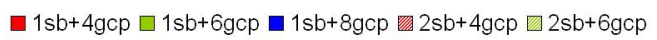
圆 $2 s b+8 g c p$ 圆3sb+4gcp 圆3sb+6gcp 圆3sb+8gcp

Figure 3: Accuracy of Pavia and Salou corridors for ISO with absolute and relative aerial control. 
Pavia corridor: 3 sub-blocks and 7 GCPs

\begin{tabular}{|c|c|c|c|c|c|c|c|c|}
\hline $\begin{array}{r}\text { Fast AT } \\
\text { ISO }\end{array}$ & DiSO & ISO & Fast AT & DiSO & $\begin{array}{c}\text { Fast AT } \\
\text { ISO }\end{array}$ & DiSO ISO & Fast AT & DiSO \\
\hline $\mathbf{8 8}^{107}$ & 256 & 84 & 144 & 242 & $\begin{array}{c}74 \\
\mathbf{8 0}\end{array}$ & $256 \quad 66$ & 119 & 242 \\
\hline RMSE - H (mm) & & & RMSE - I & & RMSE - H (mm) & & RMSE - V (mm) & \\
\hline
\end{tabular}

Salou corridor: 2 sub-blocks and 6 GCPs

\begin{tabular}{|c|c|c|c|c|c|c|c|c|c|c|}
\hline \multirow{2}{*}{ ISO } & \multirow[t]{2}{*}{ Fast AT } & \multirow{2}{*}{\multicolumn{2}{|c|}{ DiSO ISO }} & \multicolumn{2}{|c|}{ Fast AT } & \multirow[b]{2}{*}{ ISO } & \multirow[t]{2}{*}{ Fast AT } & \multicolumn{3}{|c|}{ Fast AT } \\
\hline & & & & & DiSO & & & DiSO & ISO & DiSO \\
\hline 52 & 81 & 108 & 28 & 93 & 165 & 47 & 70 & 108 & 47 & 165 \\
\hline & RMSE - H (mm) & & & RMSE - V & & & RMSE - H (mm) & & & \\
\hline
\end{tabular}

Table 5: RMSE of ChPs of selected P-C and S-C configurations.

results and as we remark in (Blázquez and Colomina, 2012a) and section 3.2, the geometries of 2 or 3 sub-blocks with only 4 GCPs or 3 sub-blocks with less than 8 GCPs are weaker than the selected geometries for Fast AT. Moreover, as the GCP redundancy numbers depend on the stochastic model, the GCP redundancy numbers for relative aerial control are higher than for absolute aerial control proving the idea pointed in (Blázquez and Colomina, 2012b) about the correctness of the stochastic model for relative aerial control. Without a complete study of the corridor Fast AT geometries it is risky to provide thresholds to accept or not a Fast AT configuration from the GCP redundancy numbers. However, in general terms, for absolute and relative Fast AT, it seems that a GCP redundancy number in the height component lower than $20 \%$ and $10 \%$ for Leica RC30 and Z/I DMC, respectivaly, indicates a weak Fast AT geometry for corridor aerial mapping.

\subsection{Fast AT results: corridor vs block aerial mapping}

In order to illustrate the performance of the Fast AT method for corridor aerial mapping against the performance of Fast AT method for block aerial mapping, the results of Pavia corridor are compared to the results of Pavia block. While the selected geometry for the Pavia corridor is a 3 sub-block configuration, the Pavia block geometry is a 1 sub-block configuration (Blázquez and Colomina, 2012a). On the other hand, although the GCP configuration is the same for both data sets, the number of image coordinates are different: $79 \times 2$ image coordinates for Pavia block against $28 \times 2$ for Pavia corridor. Figure 4 represents the configurations of both data sets.

Table 7 provides the ground accuracy results for absolute and relative Fast AT and absolute and relative ISO for Pavia corridor and Pavia block. If we compare the Pavia block and Pavia corridor results, as it was expected, the RMSE of ChPs of Pavia corridor are worse than the RMSE of ChPs of Pavia block. In average, Pavia block performance improves the Pavia corridor performance by $206 \%$. The Pavia block ground accuracy is $212 \%$ and $201 \%$ better than the Pavia corridor ground accuracy for ISO and Fast AT methods, respectively. If we compare now, the DiSO, Fast AT and ISO results, the Fast AT Pavia corridor average improvement

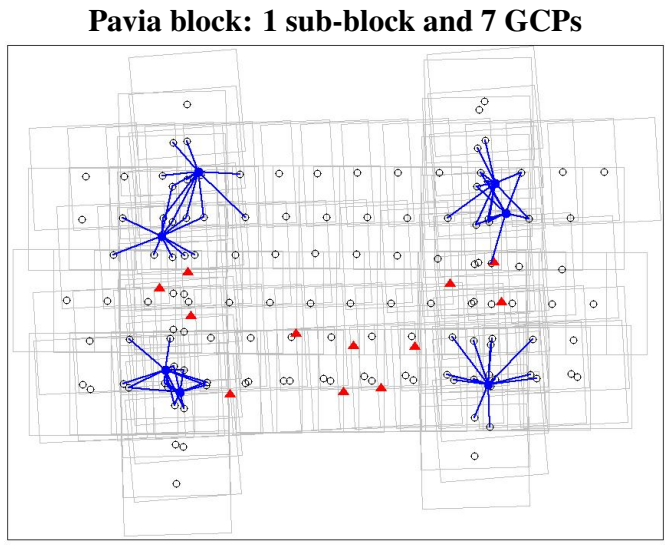

Pavia corridor:3 sub-blocks and 7 GCPs

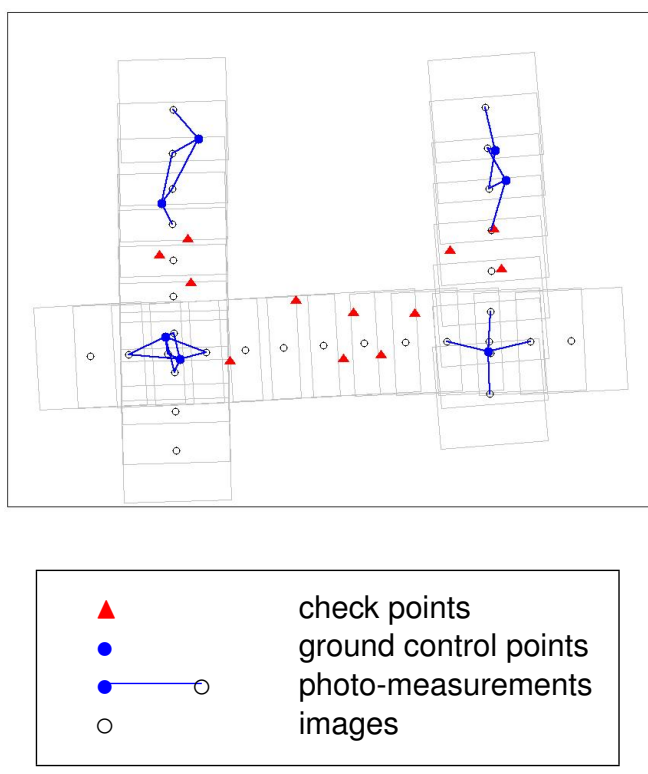

Figure 4: Pavia block and Pavia corridor layouts. 


\begin{tabular}{|c|c|c|c|c|c|c|c|c|c|c|c|c|c|c|c|c|c|c|}
\hline \multirow{2}{*}{$\begin{array}{r}\text { Aerial } \\
\text { Control } \\
\end{array}$} & \multicolumn{6}{|c|}{$\begin{array}{l}\text { Pavia corridor } \\
5 \text { GCPs }\end{array}$} & \multicolumn{3}{|c|}{7 GPCs } & \multicolumn{3}{|c|}{4 GCPs } & \multicolumn{3}{|c|}{$\begin{array}{c}\text { Salou corridor } \\
6 \mathrm{GCPs}\end{array}$} & \multicolumn{3}{|c|}{8 GPCs } \\
\hline & $r_{E}$ & $r_{N}$ & $r_{h}$ & $r_{E}$ & $r_{N}$ & $r_{h}$ & $r_{E}$ & $r_{N}$ & $r_{h}$ & $r_{E}$ & $r_{N}$ & $r_{h}$ & $r_{E}$ & $r_{N}$ & $r_{h}$ & $r_{E}$ & $r_{N}$ & $r_{h}$ \\
\hline $\mathrm{a} 1 \mathrm{sb}$ & 29 & 36 & 24 & 38 & 43 & 27 & 48 & 51 & 33 & 31 & 31 & 12 & 38 & 38 & 13 & 44 & 45 & 16 \\
\hline a 2sb & 28 & 32 & 10 & 34 & 37 & 21 & 45 & 48 & 29 & 28 & 28 & 4 & 34 & 33 & 12 & 41 & 42 & 15 \\
\hline a $3 \mathrm{sb}$ & 28 & 32 & 10 & 32 & 35 & 14 & 45 & 47 & 25 & 28 & 28 & 4 & 29 & 31 & 6 & 40 & 41 & 12 \\
\hline $\mathrm{r} 1 \mathrm{sb}$ & 44 & 49 & 52 & 54 & 57 & 58 & 67 & 67 & 64 & 47 & 48 & 43 & 58 & 57 & 50 & 72 & 71 & 57 \\
\hline r $2 s b$ & 43 & 43 & 3 & 53 & 52 & 41 & 65 & 65 & 52 & 46 & 37 & 6 & 56 & 56 & 42 & 71 & 70 & 51 \\
\hline r 3sb & 43 & 43 & 3 & 51 & 50 & 17 & 65 & 65 & 34 & 46 & 37 & 6 & 54 & 54 & 21 & 71 & 70 & 40 \\
\hline
\end{tabular}

a: absolute aerial control; r: relative aerial control.

sb: sub-block.

Table 6: GCP redundancy numbers (\%) of Fast AT for P-C and S-C depending on the sub-block and GCP configurations.

\begin{tabular}{lcccc} 
Block & \multicolumn{2}{c}{ Pavia block } & \multicolumn{2}{c}{ Pavia corridor } \\
Configuration & $R_{H}$ & $R_{V}$ & $R_{H}$ & $R_{V}$ \\
\hline DiSO & 256 & 242 & 256 & 242 \\
Absolute ISO & 39 & 40 & 107 & 84 \\
Relative ISO & 40 & 37 & 74 & 66 \\
Absolute Fast AT & 51 & 55 & 88 & 144 \\
Relative Fast AT & 45 & 63 & 80 & 119 \\
\hline
\end{tabular}

Table 7: RMSE (mm) of ChPs of selected Pavia block and Pavia corridor configurations.

factor is $86 \%$, while the average improvement factor of Fast AT Pavia block is $93 \%$. These results are consistent with the conclusions of (Blázquez and Colomina, 2012a) with respect to the performance of Fast AT method for block aerial mapping (Fast AT improves the ground accuracy of DiSO by $90 \%$ as compared to ISO) and the conclusions of the previous subsection respect to the performance of Fast AT method for corridor aerial mapping (Fast AT improves the ground accuracy of DiSO by $74 \%$ compared to ISO).

\section{CONCLUSIONS}

In this paper we review the concept and geometry of the proposed Fast AT orientation procedure and we validate this method for the corridor aerial mapping application. This paper is not an exhaustive study on the geometry and performance of Fast AT for corridor aerial mapping, but it presents the first results for this application.

Fast AT is a particular case of ISO where all the TPs are GCPs and there are few of them; i. e., Fast AT is an ISO adjustment characterized by the use of INS/GNSS-derived position and attitude, coordinates of few GCPs and image coordinates of these few GCPs. Therefore to perform Fast AT instead of the traditional ISO, the orientation and calibration lines do not need to be modified. The Fast AT practitioners only need to measure less observations.

This simple method was validated in (Blázquez and Colomina, 2012a) for block aerial mapping and the results were that Fast AT improved the ground accuracy of DiSO by $90 \%$ as compared to ISO. In this paper we test the Fast AT method for corridor aerial mapping with two different corridors and the results are that Fast AT improves the ground accuracy of DiSO by $74 \%$. Although the results for corridor aerial mapping are not so good as the Fast AT results for block aerial mapping, the performance of Fast AT corridor is still excelent. Moreover, as in the case of block aerial mapping the GCP redundancy numbers are correlated with the ground accuracy results and they can be used as a self-diagnosis tool to detect weak Fast AT geometries.

\section{REFERENCES}

Blázquez, M. and Colomina, I., 2012a. Fast AT: a simple procedure for quasi direct orientation. ISPRS Journal of Photogrammetry and Remote Sensing 71(1), pp. 1-11.

Blázquez, M. and Colomina, I., 2012b. Relative INS/GNSS aerial control in integrated sensor orientation: models and performance. ISPRS Journal of Photogrammetry and Remote Sensing 67(1), pp. 120-133.

Colomina, I., Blázquez, M., Navarro, J. A. and Sastre, J., 2012. The need and keys for a new generation network adjustment software. In: International Archives of Photogrammetry, Remote Sensing and Spatial Information Sciences, Melbourne, Australia.

Heipke, C., Jacobsen, K. and Wegmann, H., 2002. Integrated sensor orientation: test report and workshop proceedings. In: OEEPE Official Publication, Vol. 43, pp. 31-49.

Ip, A., El-Sheimy, N. and Mostafa, M., 2007. Performance analysis of Integrated Sensor Orientation. Photogrammetric Engineering and Remote Sensing 73(1), pp. 89-92.

Schwarz, K., Chapman, M., Cannon, M. and Gong, P., 1993. An integrated INS/GPS approach to the georeferencing of remotely sensed data. Photogrammetric Engineering and Remote Sensing 59(11), pp. 1667-1674.

\section{AKNOWLEDGEMENTS}

The data sets Pavia and Salou were provided by Prof. Vittorio Casella (Università di Pavia, Italy) and Josep L. Colomer (Institut Cartogràfic de Catalunya, Spain), respectively. Many thanks for these contributions. The bundle block adjustments to test Fast AT are performed with the Generic Extensible Network Approach (GENA) software platform (Colomina et al., 2012) from GeoNumerics (Barcelona, Spain). This support to our research is greatly appreciated. 